\title{
Robust control of SISO systems subject to hard input constraints
}

\author{
Wolfgang Reinelt \\ Massimo Canale \\ Division of Automatic Control \\ Department of Electrical Engineering \\ Linköpings universitet, SE-581 83 Linköping, Sweden \\ WWW: http://www. control.isy.liu.se \\ Email: wolle@isy.liu.se
}

May 2001

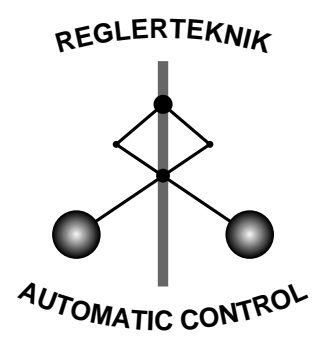

LINKÖPING

Report No.: LiTH-ISY-R-2349

Submitted to ECC 2001, Porto, Portugal

Technical reports from the Automatic Control group in Linköping are available by anonymous ftp at the address ftp.control.isy.liu.se. This report is contained in the file 2349.pdf. 


\title{
ROBUST CONTROL OF SISO SYSTEMS SUBJECT TO HARD INPUT CONSTRAINTS ${ }^{1}$
}

\author{
Wolfgang Reinelt ${ }^{a}$ and Massimo Canale ${ }^{b}$ \\ ${ }^{a}$ Department of Electrical Engineering, Linköping University, 58183 Linköping, Sweden. E-mail: wol le@isy . liu . se \\ ${ }^{b}$ Dipartimento di Automatica e Informatica, Politecnico di Torino, 10129 Torino, Italy, E-mail: canale@polito. it
}

Keywords: Constraint Control, Saturation Avoidance, Hard Bounds, Robust Control, Double Youla Parameterisation.

\begin{abstract}
The design of robust controllers that guarantee prescribed hard bounds on the control signal when facing model uncertainty is considered. Our approach to avoid a conservative design is to describe the external signals quite accuratly by means of "admissible sets", hard bounded in amplitude and rate. The core problem to solve is the calculation of the maximum output amplitude of a set of systems. This problem will be solved for a certain parameterisation of the set in a computationally attractive way. Controller design can then be formulated by employing a double Youla parameterisation of uncertain plant and stabilising controllers. We demonstrate, how the employed model uncertainty can be identified by means of standard identification techniques. Moreover, we present a combination with an MPC scheme, that allows us to obtain faster time responses and invites the possibility to encounter for time domain and frequency domain performance specifications.
\end{abstract}

\section{Introduction and Motivation}

Most practical control problems are dominated by hard bounds. Valves can only be operated between fully open and fully closed, pumps and compressors have a finite throughput capacity and tanks can only hold a certain volume. These inputor actuator-bounds convert the linear model into a nonlinear one. Exceeding these prescribed bounds causes unexpected behaviour of the system - large overshoots, low performance or (in the worst case) instability. Solving control problems subject to hard bounds, we need to restrict the amplitudes of the external signals (i.e. the reference signals) as well. In this approach, we regard reference signals, bounded in amplitude and rate (i.e. the first derivative has to be bounded in its amplitude). This appears in many systems. For example in a tank, not only the liquid-level is bounded (by the tanks height), additionally the liquid cannot change its level arbitrarily fast.

Design of controllers for systems with hard constraints is a quite vivid area of research, see for example $[1,12,15]$.

\footnotetext{
${ }^{1}$ The work was partly supported by the European Commission through the program Training and Mobility of Researchers - Research Networks and through the project System Identification (W. Reinelt) and by funds of MURST under the Project "Robustness techniques for control of uncertain systems" (M. Canale). Contacts with the participants in the European Research Network System Identification (ERNSI) are gratefully acknowledged.
}

Controller designs that encounter the saturation effect a-priori are usually splitted into two categories: (1) designs that prevent saturation of the control signal and therefore enjoy a linear framework (as long as plant and controller are linear) and (2) methods that allow saturation and are therefore facing a nonlinear setup. In the second case, analysis (in terms of stability, controllability and feasibility) of this nonlinear system is discussed in $[5,13,14]$. Design schemes that handle saturations using a nonlinear controller have been proposed [11,3].

This work clearly employs the first - saturation avoiding - philosophy. To solve the constraint control problem in a linear framework, one implicitly has to restrict the amplitude of all external signals; independent of the technique used in particular. Our approach, however, makes a further step by imposing an additional restriction on the rate of the external signals. In many practical situations, this is a more accurate description (than without rate restriction) of all external signals, possibly arising during runtime. A design, directly based on this description will avoid a conservative control system.

In this work we consider the design of a robust controllers that guarantee prescribed hard bounds on the control signal when facing an uncertain model. This is a clear progress compared to our previous efforts [7, 9], that guarantee hard bounds only in the case that the model matches the plant exactly. The core problem for the lack of robustness so far was that it was not well understood what the maximum output amplitude of a set of systems is. This problem will be solved in this work for certain parameterisation of the set in a computationally attractive way. A drawback of the saturation avoiding techniques may be a lower performance, compared to a nonlinear, gain scheduling control law for instance. It has already been pointed out above that our approach is less conservative due to a more accurate description of all possible external signals. Moreover, we present a combination with an MPC scheme, that allows us to obtain faster time responses and allows the possibility to encounter for time domain and frequency domain specifications.

\section{Maximum Output of an Uncertain System}

Before turning to actual design techniques, we need to understand how a set of systems reacts (in terms of maximum possible output amplitude) to a set of so called admissible input signals. Having solved this problem, we will be able to apply the design philosophy from previous works, which calculates/adapts [10, 9, 7] a controller, based on the exact computation of the maximum possible control signal within the control 
loop. We recap problem and solution for a single system, as discussed in [8], and generalise it:

Given a SISO LTI stable system, which is represented by its transfer function $\Pi(s)$ and its impulse response $\pi(t)$ respectively. The input is denoted by $\xi$, the output by $\lambda$. The following constraints hold for the continuous and piecewise differentiable input signal $\xi$ :

$$
\begin{aligned}
& |\xi(t)| \leq \Xi \\
& |\dot{\xi}(t)| \leq \dot{\Xi}
\end{aligned}
$$

for $t>0$, where $\Xi, \dot{\Xi}>0$ are given constant values and $\xi(t)=0, t \leq 0$. We call those reference signals, which fulfill eqns.(1,2) $(\Xi, \dot{\Xi})$-admissible, or short $\xi \in \mathcal{A}(\Xi, \dot{\Xi})$. We are looking for the maximum amplitude $\Lambda_{m}(t)$ of the output $\lambda$ (up to time $t$ ) for all $(\Xi, \dot{\Xi})$-admissible inputs, i.e.

$\Lambda_{m}(t)=\sup _{\xi \in \mathcal{A}(\Xi, \dot{\Xi})} \sup _{0<\tau \leq t}|\lambda(\tau)|=\sup _{\xi \in \mathcal{A}(\Xi, \dot{\Xi})} \sup _{0<\tau \leq t}|\pi(\tau) \star \xi(\tau)|$,

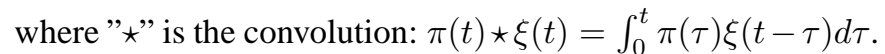
It is clear, that the function $\Lambda_{m}(t)$ is non-decreasing in time $t$.

It turns out that the solution of this problem can be approximated quite well by linear programming techniques [8]. The solution of this problem allows design or at least adaption of controllers, that ensure a prescribed hard bound on the control signal $[7,9,10]$. This prescribed bound holds, however, only in the case that the actual system and the model, used for design, are exactly the same. The core problem for the lack of robustness is that it is not well understood what the maximum output amplitude of a set of systems is. This problem will be solved now:

Suppose a set of stable LTI SISO systems:

$$
\Pi_{\theta}(s):=\Pi_{c}(s)+B(s) \cdot \theta,
$$

where $B(s)=\left[B_{1}(s), \ldots, B_{n}(s)\right]^{T}$ is a set of stable basis functions, for instance orthonormal basis functions, and $\theta=\left[\theta_{1}, \ldots, \theta_{n}\right]^{T}$ is a parameter, located in a rectangular box:

$$
\theta_{i}^{l o} \leq \theta_{i} \leq \theta_{i}^{h i}, \quad \forall i .
$$

In fact, the model set (3) is affinely parameterised in uncertainty, given by (4). Let the input signal $\xi$ obey the same constraints $(1,2)$ as above. According to [8], finding the maximum output amplitude of system (3) can be solved via Quadratic Programming (QP), when looking at a discretised version:

$$
\Lambda_{m}=\sup _{\xi_{o, k}} \sum_{k=0}^{\infty} \pi_{\theta, k} \xi_{k}
$$

where $\left\{\pi_{\theta, k}\right\}$ is the impulse response of system $\Pi_{\theta}$ in (3), and $\xi_{k}$ is a (time inverted) admissible input signal, which can be (approximately) described for the discrete time case by

$$
\begin{array}{ll}
-\Xi \leq \quad \xi_{o, k} & \leq \Xi, \quad \forall k \geq 0, \\
-\dot{\Xi} \leq & \frac{\xi_{o, k+1}-\xi_{o, k}}{t_{k+1}-t_{k}} \quad \leq \dot{\Xi}, \quad \forall k \geq 0 .
\end{array}
$$

The core problem, to be solved on a finite time grid of size, say, $N+1$ is then:

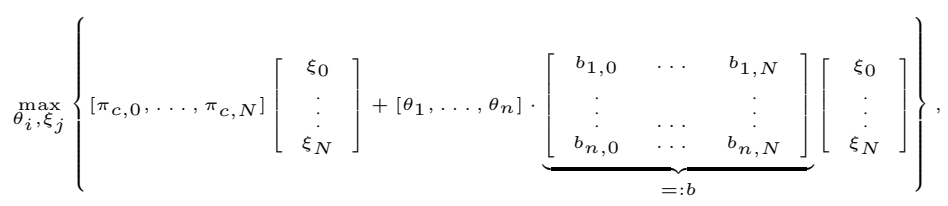

where $\left[\pi_{c, 0}, \ldots, \pi_{c, N}\right]$ is the impulse response of $\Pi_{c}$, evaluated at the first $N+1$ time stamps, and $b_{i, 0}, \ldots, b_{i, N}$ is the impulse response of basis function $B_{i}$, evaluated on the same time grid. Note that they are known. The restrictions on $\theta_{i}$ and $\xi_{j}$ are given by $(6,7,4)$, which are obviously linear. Denoting $\xi=\left[\xi_{0}, \ldots, \xi_{N}\right]^{T}, \pi_{c}=\left[\pi_{c, 0}, \ldots, \pi_{c, N}\right]$ and adding some obvious zeros blocks, the above maximisation becomes:

$$
\max _{\theta_{i}, \xi_{j}}\left\{\left[0, \pi_{c}\right]\left[\begin{array}{l}
\theta \\
\xi
\end{array}\right]+\left[\begin{array}{ll}
\theta^{T} & \xi^{T}
\end{array}\right]\left[\begin{array}{ll}
0 & b \\
0 & 0
\end{array}\right]\left[\begin{array}{l}
\theta \\
\xi
\end{array}\right]\right\}
$$

subject to

$$
\begin{aligned}
& \theta_{i}^{l o} \leq \quad \theta_{i} \quad \leq \theta_{i}^{h i}, \quad \forall i . \\
& -\Xi \leq \quad \xi_{o, k} \quad \leq \Xi, \quad \forall k \geq 0, \\
& -\dot{\Xi} \leq \frac{\xi_{o, k+1}-\xi_{o, k}}{t_{k+1}-t_{k}} \quad \leq \dot{\Xi}, \quad \forall k \geq 0 \text {. }
\end{aligned}
$$

Optimising (8) subject to $(9,10,11)$ is obviously a QP problem. Moreover we observe that the description of the model uncertainty in (9) can easily replaced by any other linear set of parameters (parallelotopes for instance) without changing the character of the optimisation problem.

\section{Controller Design}

We will now link the result from Sec. 2 to a controller design. Suppose a controller $C_{\text {nom }}$ stabilising the nominal plant $G_{n o m}$, is given, and assume moreover normalised right coprime factorisations of them:

$$
\begin{aligned}
G_{\text {nom }} & =N_{G} \cdot D_{G}^{-1} \\
C_{\text {nom }} & =N_{C} \cdot D_{C}^{-1} .
\end{aligned}
$$

Suppose now a so-called double Youla parameterisation $[4,16]$ of uncertain plant and a set of stabilising controllers in terms of uncertainty in the coprime factors, cf. Fig. 1:

$$
\begin{aligned}
G_{\Delta} & :=\left(N_{G}+D_{C} \Delta_{G}\right)\left(D_{G}-N_{C} \Delta_{G}\right)^{-1} \\
C_{\Delta} & :=\left(N_{C}+D_{G} \Delta_{C}\right)\left(D_{C}-N_{G} \Delta_{C}\right)^{-1}
\end{aligned}
$$

All in all, the above two sets parameterise a set of controllers, that stabilise an uncertain plant:

3.1 Theorem ([4]) Given a controller $C_{n o m}=N_{C} \cdot D_{C}^{-1}$ that internally stabilises the plant $G_{n o m}=N_{G} \cdot D_{G}^{-1}$, where $N_{x}, D_{x}$ are normalised right coprime factors. Then the set of controllers $C_{\Delta}$ as described in (15) stabilises the uncertain plant $G_{\Delta}$ as described in (14), if

$$
\left\|\Delta_{C}\right\|_{\infty}\left\|\Delta_{G}\right\|_{\infty}<1
$$




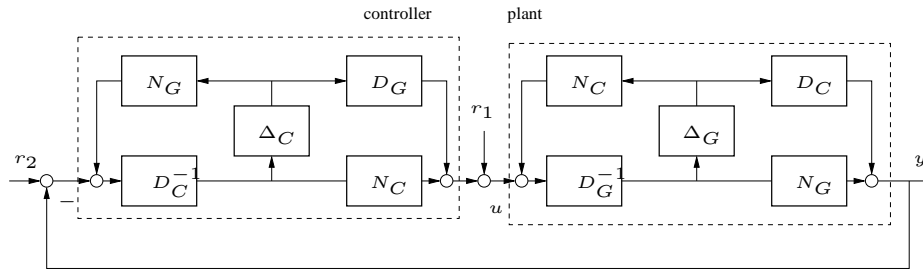

Figure 1: Double Youla parameterisation of uncertain plant and (sub)set of stabilising controllers.

We note, that the above condition is only sufficient and not necessary. The introduction of weights may reduce the amount of conservatism, as (16) is a relation between the $\mathcal{H}_{\infty}$ norms rather than a frequency-by-frequency condition.

Our aim is now to calculate the maximum amplitude of the control signal in an (uncertain) control system, with respect to the amplitude and rate of the reference signal. Now, the transfer function from reference signal to control signal is, as a function of the two uncertainties in controller and plant:

$$
\begin{aligned}
T_{r_{2} u} & =T_{r_{2} u}\left(\Delta_{G}, \Delta_{C}\right)=\frac{C_{\Delta}}{1+C_{\Delta} G_{\Delta}}= \\
& \frac{1-N_{C} D_{G}^{-1} \Delta_{G}+D_{G} N_{C}^{-1} \Delta_{C}-\Delta_{G} \Delta_{C}}{\left(G_{n o m}+C_{n o m}^{-1}\right)\left(1+\Delta_{C} \Delta_{G}\right)}
\end{aligned}
$$

whenever this expression exists. Obviously, (18) is affine in either of the $\Delta$ 's, whenever the other is zero. In this case, the Quadratic Program in Sec. 2 with $\Pi=T_{r_{2} u}, \xi=r_{2}, \lambda=u$ can be used to establish the maximum possible amplitude of the control signal, whenever the uncertainty $\Delta$ obeys $\Delta_{G}=B_{G} \theta_{G}$ and $\Delta_{C}=B_{C} \theta_{C}$ respectively, where $\theta_{G}, \theta_{C}$ restricted as in (4). We postpone this calculation for a given $\Delta_{G}$ to Sec. 4 and assume in the remainder of this section, that we are able to estimate/identify $\Delta_{G}=B_{G} \theta_{G}$ along with a linear constraint on $\theta_{G}$ for a given nominal setup $G_{n o m}, C_{n o m}$.

We now propose an algorithm for controller design, guaranteeing a control signal less then a desired bound $u^{\text {des }}$ :

\subsection{Algorithm (first approach)}

1. Get i/o data, estimate $G_{n o m}=N_{G} D_{G}^{-1}$.

2. Get stabilising controller $C_{n o m}=N_{C} D_{C}^{-1}$.

3. Estimate the coprime factor error $\Delta_{G}$, using $G_{n o m}, C_{n o m}$ and the closed loop data record $\left(r_{1}, u, y\right)$.

4. Calculate maximum control signal $u_{\max }$, applying the QP to $T_{r_{2} u}\left(\Delta_{G}, 0, C_{n o m}\right), \forall \Delta_{G}$ :

- $u_{\max } \leq u^{\text {des }}$ : ready!

- $u_{\max }>u^{\text {des}}$ : Pick another stabilising controller $C^{*}$, let $C_{n o m}=C^{*}$ and goto step 3 .

We note that after failure, we have to re-identify the model uncertainty in terms of the coprime factor $\Delta_{G}$, based on a new data record. This is due to the fact, that expression (18) is only affine in $\Delta_{G}$, when $\Delta_{C}=0$.

An optional pre-check can be added after step 2: Get all con$\rightarrow$ trollers (or a reasonable subset, with e.g. restrictions on order) satisfying (16), and calculate the maximum control signals for the nominal plant and all controllers (i.e. $\Delta_{G}=0$ in (18)). If their minimum is larger than the desired one, there is no solution using this approach. Otherwise, all controllers producing a smaller control signals are candidates when proceeding.

We state a variant of the above algorithm, which is intended to decrease the amount of re-calculations of $\Delta_{G}$.

\subsection{Algorithm (second approach)}

1. Get i/o data, estimate $G_{n o m}=N_{G} D_{G}^{-1}$.

2. Get stabilizing controller $C_{n o m}=N_{C} D_{C}^{-1}$.

3. Estimate the coprime factor error $\Delta_{G}$, using $G_{n o m}, C_{n o m}$ and the closed loop data record $\left(r_{1}, u, y\right)$.

4. Calculate maximum control signal $u_{\max }$, apply the QP to $T_{r_{2} u}\left(\Delta_{G}, 0, C_{n o m}\right), \forall \Delta_{G}$ :

- $u_{\max } \leq u^{\text {des }}$ : ready!

- $u_{\max }>u^{\text {des}}$ : Pick a stab controller $C^{*}=$ $\left(N_{C}+D_{G} \Delta_{C^{*}}\right)\left(D_{C}-N_{G} \Delta_{C^{*}}\right)^{-1}$ according to $\left\|\Delta_{C^{*}}\right\|_{\infty}\left\|\Delta_{G}\right\|_{\infty}<\epsilon \leq 1$

5. Calculate maximum control signal $u_{\max }$, apply the QP to $\tilde{T}_{r u}\left(\Delta_{G}, \Delta_{C^{*}}, C_{n o m}\right), \forall \Delta_{G}$ :

$\tilde{T}_{r u}\left(\Delta_{G}, \Delta_{C^{*}}, C_{n o m}\right)$

$\frac{1-N_{C} D_{G}^{-1} \Delta_{G}+D_{G} N_{C}^{-1} \Delta_{C^{*}}-\Delta_{G} \Delta_{C^{*}}}{\left(G_{\text {nom }}+C_{\text {nom }}^{-1}\right)(1 \pm \epsilon)}$

- $u_{\max } \leq u^{\text {des }}$ : replace $C_{\text {nom }}$ by $C^{*}$, return to step 3 .

$\bullet u_{\max }>u^{\text {des}}$ : pick another $\Delta_{C^{*}}$, goto step 5 .

3.4 Remark In the algorithms stated above, a simple check if the "new" nominal control loop obeys the desired bound on the control signal, will be of advantage, as it is a necessary condition for the new/modified controller to succeed. We observe, that step 5 of the second algorithm looks for the controller in the $\epsilon$-ball around the nominal one, that has a sufficiently small control signal. This search for a "minimal" $C_{\Delta}$ is quite similar to the techniques used in [10], where nonlinear optimisation is applied successfully.

\section{Identification of the uncertain plant}

In order to apply the design procedure outlined in the previous sections, an uncertainty model of the system to be controlled has to be estimated in terms of a nominal model $G_{n o m}$ together with the "joint uncertainty" in the coprime factors. This is known from literature, see for instance [17, Sec.5.4]. We repeat the necessary technicalities and state them as: 


\subsection{Algorithm (Identification of $\Delta_{G}$ )}

1. Given nominal plant and controller $G_{n o m}, C_{n o m}$ and the closed loop data record $\left(r_{1}, u, y\right)$.

2. Calculate the filtered residuum: $z=\left(D_{c}+\right.$ $\left.G_{n o m} N_{c}\right)^{-1}\left(y-G_{n o m} u\right)$.

3. Identify $\Delta_{G}$ from the setup: $z=\Delta_{G} \cdot r_{1}$, assuming $\Delta_{G}=$ $B_{G} \theta_{G}$ and $\theta_{G}$ linearly restricted as in (4).

4.2 Remark Our final aim is a closed loop system that contains a saturation nonlinearity. Hence, in order to apply linear techniques in identification, the amplitude of the input signal has to satisfy the input constraints (which is, in this context, the search for a proper reference signal $r_{1}$ ). Saying this, we implicitly assume the knowledge of the saturation level (which we do as well for the controller design).

4.3 Remark To make the framework consistent, the controller designed in step 2 has to stabilize the "real plant" (meaning the identified model set in this context). This, however, implies that the open loop experiment performed in step 1 of both algorithms has to deliver a model set for the plant to be controlled in order to allow a robust controller design.

\section{Performance Enhancement using MPC}

The design procedure proposed in the previous sections can be suitably used in order to enhance the time response performances and to couple in an effective way both time and frequency domain specifications. In a time domain design context, the satisfaction of hard input constraint can be achieved, for the nominal model $G_{n o m}$, by computing the control moves $\bar{u}$, in a receding horizon fashion by solving (e.g.) the following optimisation problem at each time step:

$$
\begin{aligned}
& \min _{U}\left\{\sum_{i=1}^{h_{p}}\|y(k+i \mid k)-r\|^{2}+\sum_{i=0}^{h_{c}}\|\bar{u}(k+i \mid k)\|^{2}\right\} \\
& U=\left[\bar{u}(k \mid k), \ldots, \bar{u}\left(k+h_{c}-1 \mid k\right)\right] \\
& \text { s.t. } U \in \mathcal{U}=\left\{\bar{u} \in U: \bar{u}_{\min } \leq \bar{u} \leq \bar{u}_{\max }\right\}
\end{aligned}
$$

where $\bar{u}_{\max }>0>\bar{u}_{\min }, r$ is a prescribed reference signal, $\|\cdot\|$ is the Euclidean norm and $h_{p}$ and $h_{c}$ are respectively the prediction and the control horizons satisfying $h_{c}<h_{p} \leq \infty$ (see e.g. the recent survey [6]). Now consider the control structure depicted on Fig. 2 where the controller $C_{n o m}$ can be designed using the procedure outlined in the previous sections, the command $\bar{u}$ is computed according to (19) and $\bar{y}$ is the output of model $G_{n o m}$ for the input $\bar{u}$ (i.e. $\bar{y}=G_{n o m} \bar{u}$ ).

This way the system input $u$ which has to be bounded is the sum of the two components $\bar{u}$ (the MPC one) and $\delta u$ (the contribution of controller $C_{\Delta}$ ):

$$
u=\bar{u}+\delta u
$$

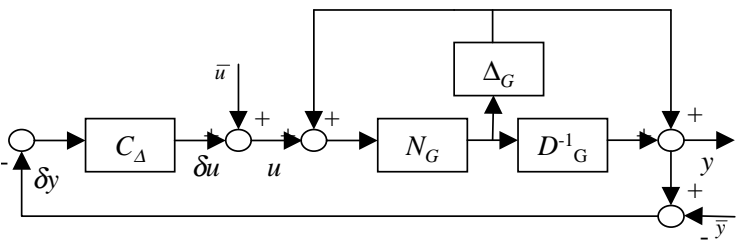

Figure 2: Enhanced control structure

It is easy to verify that the control structure in Fig. 2 is equivalent to the one represented in Fig. 1 by posing: $r_{1}=\bar{u}$ and $r_{2}=\bar{y}$. It has to be noted that, the application of such signals, produces as an effect the control loop in Fig. 1 to track the "nominal trajectory" $\bar{y}$. It is easy to verify that the relation between the command signal $u$ and input $\bar{u}$ is given by:

$$
u=\frac{G_{n o m}+C_{\text {nom }}^{-1}-D_{G}^{-1}\left(D_{C}+N_{C} N_{G} D_{G}^{-1}\right) \Delta_{G}}{\left(G_{\text {nom }}+C_{\text {nom }}^{-1}\right)\left(1+\Delta_{G} \Delta_{C}\right)} \bar{u}
$$

which, for $\Delta_{C}=0$, is affine in $\Delta_{G}$. In this case (21) can be rewritten as:

$$
u=\left(1-\frac{D_{G}^{-1}\left(D_{C}+N_{C} N_{G} D_{G}^{-1}\right) \Delta_{G}}{\left(G_{\text {nom }}+C_{\text {nom }}^{-1}\right)}\right) \bar{u}
$$

This way it results

$$
\delta u=-\frac{D_{G}^{-1}\left(D_{C}+N_{C} N_{G} D_{G}^{-1}\right) \Delta_{G}}{\left(G_{n o m}+C_{n o m}^{-1}\right)} \bar{u}
$$

Now, in order to satisfy the command limitation, the two components $\bar{u}$ and $\delta u$ may be designed such that, for a $0 \leq \varepsilon \leq 1$ :

$$
\begin{aligned}
& |\delta u| \leq \varepsilon \cdot u^{\text {des }},|\bar{u}| \leq(1-\varepsilon) \cdot u^{\text {des }} \\
& \Longrightarrow|u|=|\bar{u}+\delta u| \leq|\bar{u}|+|\delta u| \leq u^{\text {des }}
\end{aligned}
$$

The limitation on $\bar{u}$ can be achieved by a standard MPC design, whereas, for the limitation on $\delta u$, the constraint control procedure outlined in the previous sections can be exploited.

\section{Example}

The proposed constrained control procedure has been applied to the system considered in [2] whose transfer function is:

$$
\begin{aligned}
& G(s)= \\
& \frac{0.001 s^{5}+0.02 s^{4}+0.11 s^{3}+0.32 s^{2}+0.49 s+0.29}{s^{6}+0.50 s^{5}+4.59 s^{4}+1.37 s^{3}+5.22 s^{2}+0.55 s+0.07}
\end{aligned}
$$

The design procedure has been worked out employing a $4^{\text {th }}$ order identified nominal model with transfer function:

$$
G_{n o m}(s)=\frac{-0.0852 s^{3}+0.0079 s^{2}+0.2008 s+0.125}{s^{4}+0.1864 s^{3}+2.19 s^{2}+0.2355 s+0.0311}
$$

In Fig. 3 the Bode plots of $G$ and $G_{n o m}$ are reported. Both time and frequency domain specifications have been taken into 

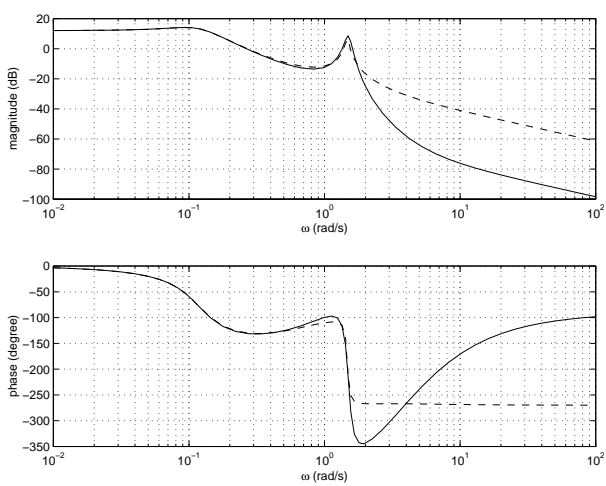

Figure 3: Bode diagrams for systems $G$ (solid) and model $G_{\text {nom }}$ (dashed).

account. In particular, a hard constraint on the control variable $|u| \leq u^{\text {des }}=0.4$ for reference signals satisfying $R_{2}=1$ and $\dot{R}_{2}=1$ together with a low frequency limitation on the nominal sensitivity function $S=\frac{1}{1+G_{n o m} C_{n o m}}$ such that $|S(j \omega)| \leq 0.1$ for $0 \leq \omega \leq 0.01 \mathrm{rad} / \mathrm{s}$ have to be satisfied. In order to meet the prescribed requirements, together with robust stability in face of the additive model uncertainty found in [2], Alg. 3.2 has been applied. The input signal $r_{1}$ used for the identification of $\Delta_{G}$ has been obtained by means of the idinput routine of Matlab Identification Toolbox exciting frequencies up to $5 \mathrm{rad} / \mathrm{s}$ plus a step signal (detecting the steady state component). In order to avoid saturation of the control variable when collecting data, nominal simulations have been carried out to tune the amplitude of $r_{1}$ in order to match hard input constraints on $u$. In Fig. 4, signal $r_{1}$ is reported together with the corresponding $u$. In order to reduce the complexity in
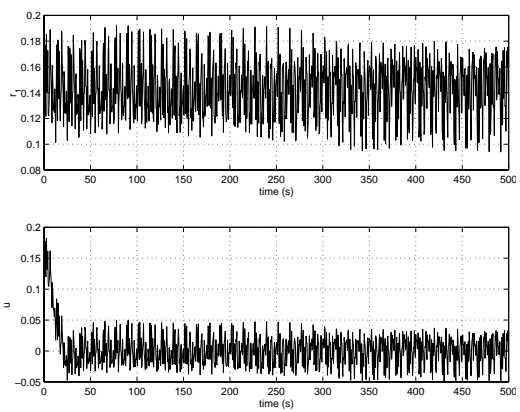

Figure 4: Signals employed in identification of $\Delta_{G}$. Above: $r_{1}$. Below: $u$.

the maximal output computation, a second order Kautz basis has been chosen as parameterization for $\Delta_{G}$. The Kautz basis parameters have been selected using the poles of the $2^{\text {nd }}$ order rough output error model obtained by the oe routine of Matlab Identification Toolbox using the collected data (i.e. $r_{1}$ and the filtered residuum $z$ ). To guarantee robust stability in face of the identified open loop model uncertainty (see Remark 4.3), standard $\mathcal{H}_{\infty}$ techniques have been used for controller design solving the optimisation problem:

$$
\begin{aligned}
& C_{n o m, C C}=\arg \min _{C}\left\|\frac{W_{S}}{1+G_{n o m} C}\right\|_{\infty} \\
& \text { s.t. }\left\|\frac{W_{E} \cdot C}{1+G_{n o m} C}\right\|_{\infty}=\left\|W_{E} T_{r_{2} u, n o m}\right\|_{\infty}<1
\end{aligned}
$$

where $W_{S}$ is a suitable frequency dependent weighting function used to shape the nominal sensitivity function and $W_{E}$ is a rational low order function overbounding the model uncertainty as tight as possible (for details see [2]). In this context, lower values on the control variable can be obtained by appropriately penalising the function $T_{r_{2} u, n o m}$. To compute the maximal control amplitude when applying $C_{n o m, C C}$, we recall (18) which, for $\Delta_{C}=0$, gives:

$$
T_{r_{2} u}=\frac{1-N_{C} D_{G}^{-1} \Delta_{G}}{G_{n o m}+C_{n o m, C C}} .
$$

Supposing $G_{n o m}$ stable and $C_{n o m, C C}$ minimum phase we can adopt the following coprime factorisations:

$$
N_{G}=G_{n o m}, D_{G}=1, N_{C}=1, D_{C}=C_{n o m, C C}^{-1}
$$

obtaining $T_{r_{2} u}=\frac{C_{n o m, C C}}{1+G_{n o m} C_{n o m, C C}}\left(1-\Delta_{G}\right)$. This way, the maximal command $u_{\max }$ can be computed as

$$
\begin{aligned}
u & =T_{r_{2} u} \cdot r_{2}=\frac{C_{n o m, C C}}{1+G_{n o m} C_{n o m, C C}}\left(1-\Delta_{G}\right) \cdot r_{2}= \\
& =\underbrace{\frac{C_{n o m, C C}}{1+G_{n o m} C_{n o m}, C C} \cdot r_{2}}_{=u_{n o m}} \underbrace{-\frac{C_{n o m, C C}}{1+G_{n o m} C_{n o m} C C} \Delta_{G} \cdot r_{2}}_{=\delta u_{n o m}}= \\
& =u_{n o m}+\delta u_{n o m}
\end{aligned}
$$

then $|u| \leq u^{\text {des }}$ because $\left|u_{\text {nom }}\right|+\left|\delta u_{\text {nom }}\right| \leq u^{\text {des }}$ (see (24)). The application of Alg. 3.2 produced a controller $C_{n o m, C C}$ :

$$
\begin{aligned}
& C_{n o m, C C}(s)= \\
& \frac{2.30 s^{5}+6.17 s^{4}+6.1 s^{3}+13.12 s^{2}+1.42 s+0.18}{s^{6}+40.76 s^{5}+22.69 s^{4}+93.49 s^{3}+37.24 s^{2}+6.16 s}
\end{aligned}
$$

with $\left|u_{\text {nom }}\right| \leq 0.31,\left|\delta u_{\text {nom }}\right| \leq 0.09$ and therefore $|u| \leq 0.4$, enabling the satisfaction of the prescribed specifications relaxing with a factor of $1.9 T_{r_{2} u, n o m}$ function. With the purpose of enhancing the performances achieved by controller $C_{n o m, C C}$, an MPC control law has been designed imposing $|\bar{u}|=0.25$ and $\varepsilon=0.37$ considering a trapezoidal reference signal $r_{2}$ with starting and final slopes of 1 and -1 respectively (thus $\left(R_{2}, \dot{R}_{2}\right)$ admissible) in the optimisation problem (19). By means of (25), a controller $C_{\text {nom, } M P C}$ was designed to satisfy $|\delta u| \leq \varepsilon \cdot u^{\text {des }}$. The computation of the maximal output can be worked out using the coprime factorisation (26), replacing $C_{n o m, C C}$ by $C_{n o m, M P C}$, and noting that (22) can be rewritten as $u=\left(1-\Delta_{G}\right) \cdot \bar{u}$, so that $\delta u=-\Delta_{G} \cdot \bar{u}$. The transfer function of the computed controller $C_{n o m, M P C}$ is:

$$
\begin{aligned}
& C_{n o m, M P C}(s)= \\
& \frac{1.61 s^{5}+4.33 s^{4}+4.28 s^{3}+9.21 s^{2}+s+0.13}{s^{6}+54.09 s^{5}+24.47 s^{4}+121.8 s^{3}+38.99 s^{2}+5.45 s}
\end{aligned}
$$


obtaining (without relaxation on $T_{r_{2} u}$ ) $|\delta u| \leq 0.14$ and so $|u| \leq|\bar{u}|+|\delta u|=0.39$. This way, using the combined action of controller $C_{n o m, M P C}$ plus the MPC control law a performance improvement has been achieved with respect to the ones obtained by the single action of the cascade controller $C_{n o m, C C}$. In Fig. 5 the frequency behaviour of the nominal sensitivity magnitude for both the designed controllers (i.e. $C_{n o m, C C}$ and $C_{n o m, M P C}$ plus MPC control law) are reported and compared showing a slight increase of the low frequency attenuation properties of the combined scheme. Moreover, in Fig. 6, the simulated time responses of the output $y$ and of the command $u$ due the trapezoidal reference $r$ are illustrated. It can be noted that the employment of the MPC control law has produced, as an effect, a faster time response together with a less overshoot on the output.

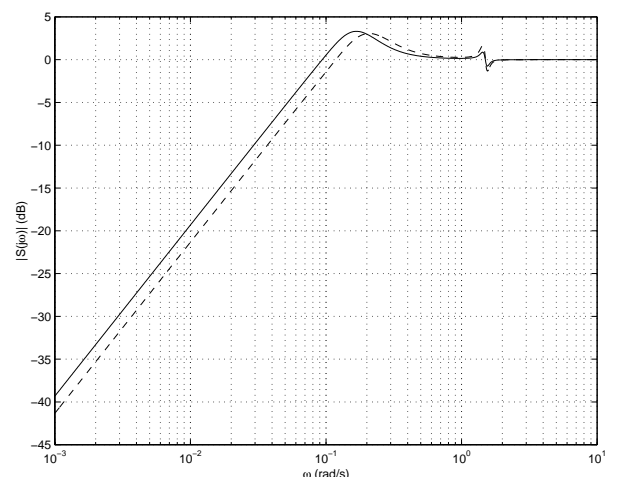

Figure 5: Nominal sensitivity magnitude (solid for controller $C_{n o m, C C}$, dashed for controller $C_{n o m, M P C}$.
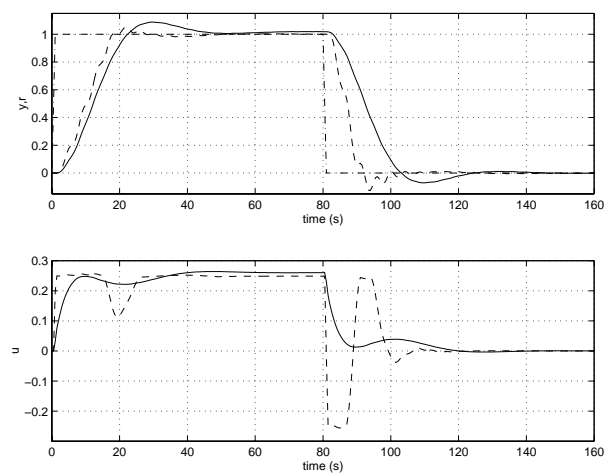

Figure 6: Above: output $y$ (solid for controller $C_{n o m, C C}$, dashed for controller $C_{n o m, M P C}$ plus MPC law) and reference $r$ (dash-dotted). Below: command $u$ (solid for controller $C_{n o m, C C}$, dashed for controller $C_{n o m, M P C}$ plus MPC law).

\section{Conclusions}

The design of robust controllers that guarantee prescribed hard bounds on the control signal when facing an uncertain model has been treated. Two aspects within our approach avoid a conservative design: (1) a more accurate description of all possible external signals in terms of "admissible" reference signals, hard bounded in amplitude and rate. (2) a combination with an MPC scheme, that allows us to obtain faster time responses and invites the possibility to encounter for time domain and frequency domain performances. While the second assumption is rather new in this line of research, the first one is at at least well studied in the "non-robust" case. The core problem for the lack of robustness so far was that it was not well understood what the maximum output amplitude of a set of systems is. This problem was solved in this work employing a (double) Youla parameterisation.

\section{References}

[1] D. S. Bernstein and A. N. Michel. Special Issue Saturating Actuators. Int. J. of Robust and Nonlinear Control, 5, 1995.

[2] M. Canale, S. A. Malan and M. Milanese. Model quality evaluation in identification for $H_{\infty}$ control. IEEE Trans. on Automatic Control, 43:1, pp.125-132, 1998.

[3] J. A. De Dona, R. Moheimani, and G. C. Goodwin. Robust combined PLC/LHG controller with allowed over-saturation of the input signal. In Proc. of the ACC, pp.750-754, Chicago, IL, USA, 2000.

[4] S. G. Douma, P. M. J. van den Hof, and O. H. Bosgra. Controller tuning freedom under plant identification uncertainty: double Youla beats gap in stability. In Proc. of the ACC, Arlington, VA, USA, June 2001.

[5] E. G. Gilbert and K. T. Tan. Linear systems with state and control constraints: The theory and applications of maximal output admissible sets. IEEE Trans. on Automatic Control, 36(9):1008-1020, Sept. 1991

[6] D. Q. Mayne, J. B. Rawlings, C. V. Rao, and P. O. M. Skokaert. Constrained model predictive control: Stability and optimality. Automatica, 36:789-814, 2000.

[7] W. Reinelt. $\mathcal{H}_{\infty}$ Loop Shaping for Systems with Hard Bounds. In Proc. of the Int Symp on QFT, Durban, South Africa, 1999.

[8] W. Reinelt. Maximum Output Amplitude of Linear Systems for certain Input Constraints. In Proc. of the CDC, Sydney, Australia, 2000.

[9] W. Reinelt. Robust Control of a Two-Mass-Spring System subject to its Input Constraints. In Proc. of the ACC, Chicago, IL, USA, 2000.

[10] W. Reinelt. Design of optimal control systems with bounded control signals. In Proc. of the ECC, Porto, Portugal, Sept. 2001.

[11] A. Saberi, J. Han, and A. A. Stoorvogel. Constrained stabilization problems for linear plants. In Proc. of the ACC, Chicago, IL, USA, 2000.

[12] A. Saberi, A. A. Stoorvogel, and P. Sannuti. Control of Linear Systems with Regulation and Input Constraints. Springer Verlag, 2000.

[13] E. D. Sontag. An algebraic approach to bounded controllability of linear systems. Int. J. of Control, 39(1):181-188, Jan. 1984.

[14] H. J. Sussmann, E. D. Sontag, and Y. Yang. A general result on the stabilization of linear systems using bounded controls. IEEE Trans. on Automatic Control, 39(12):2411-2425, Dec. 1994.

[15] S. Tabouriech and G. Garcia, editors. Control of Uncertain Systems with Bounded Inputs. Springer Verlag, London, UK, 1997.

[16] T. T. Tay, J. B. Moore, and R. Horowitz. Indirect adaptive techniques for fixed controller performance enhancement. Int. J. of Control, 50(5):1941-1959, 1989.

[17] P. M. Van den Hof. Closed loop issues in system identification. Annual Reviews in Control, 22:173-186, 1998. 